\title{
Conformal mapping approach for permanent magnet synchronous machines: on the modeling of saturation
}

\author{
MARTin Hafner, DAVId Franck, KAy HAMEyer \\ Institute of Electrical Machines - RWTH Aachen University \\ Schinkelstraße 4, D-52062 Aachen, Germany \\ e-mail: Martin.Hafner@IEM.RWTH-Aachen.de
}

(Received: 29.11.2011, revised: 10.01.2012)

\begin{abstract}
In the electromagnetic field simulation of modern servo drives, the computation of higher time and space harmonics is essential to predict torque pulsations, radial forces, ripple torques and cogging torque. Field computation by conformal mapping (CM) techniques is a time-effective method to compute the radial and tangential field components. In the standard $\mathrm{CM}$ approach, computational results of cogging torque simulations as well as overload operations observe deviations to nonlinear finite element (FE) simulations due to the neglection of slot leakage and saturation effects. This paper presents an extension of the classical CM. Additional CM parameters are computed from single finite element computations so as to consider both effects listed above in the model over a wide operation range of the electrical drive. The proposed approach is applied to a surface permanent magnet synchronous machine (SM-PMSM), and compared to numerical results obtained by finite element analysis (FEA). An accuracy similar to that of FE simulations is obtained with however the low computation time that is characteristic for analytical models.
\end{abstract}

Key words: conformal mapping, air gap permeance, magnetic field, SM-PMSM, torque computation

\section{Standard conformal mapping}

The air gap field computation by conformal mapping is generally obtained from the solution of a linear Laplace problem, assuming an infinite permeability of the magnetic core. This results in a linear time invariant system, and consequently the field excitation by magnets and coils, as well as the influence of the slotting, can be modeled individually.

Assuming a slotless stator, the field $\vec{B}(\Theta)$ at a certain coordinate angle $\Theta$ in the air gap, $\Theta \in[0,2 \pi]$, consists of a radial flux density $B_{r}(\Theta)$ and a tangential flux density $B_{\varphi}(\Theta)$

$$
\vec{B}(\Theta)=B_{r}(\Theta) \cdot \vec{e}_{r}+B_{\varphi}(\Theta) \cdot \vec{e}_{\varphi} .
$$

In classical representation, this two-dimensional vector field is represented by the complex field quantity $B(\Theta)$, defined as 


$$
\underline{B}(\Theta)=B_{r}(\Theta)+{ }_{J} B_{\varphi}(\Theta) .
$$

The complex notation is applied in this work. The angle dependent quantities $B_{r}(\Theta)$ and $B_{\varphi}(\Theta)$ can be expanded into a Fourier Series

$$
B_{x}(\Theta)=\sum_{n=1}^{\infty} a_{n, x} \cos (2 \pi n p \Theta)+\left.b_{n, x} \sin (2 \pi n p \Theta)\right|_{x=r, \varphi},
$$

where $n$ is the frequency order and $p$ the number of pole pairs. In this representation of the air gap field components, the Fourier coefficients $a_{n, x}$ and $b_{n, x}$ are the solution of a linear Laplace problem with magnets and a slotless stator, depending on the magnetization configuration [1-3]. The field at a certain instance of time $t$ due to the rotor movement is given by

$$
\underline{B}(t)=\underline{B}\left(\Theta+\omega_{r} t\right),
$$

where $\omega_{r}$ is the angular speed of the rotor. The position shifting of $\underline{B}$ due to an arbitrary instance of time $t$ is indicated by the operator in the sense of (4)

$$
\underline{B}(t)=\underline{B} \cdot e^{\imath \omega_{r} t} .
$$

Stator slotting significantly influences the magnetic field distribution. It is modeled by so called "relative permeance functions". These permeance functions $\underline{\lambda}$ represent the impact of slotting on the slotless field distribution and can be obtained by Schwarz-Christoffel transformations $[4,5]$. Correlating the field distribution with slotting, ${ }^{s} \underline{B}(t)$, with the field without slotting (5), yields the complex $\underline{\lambda}$

$$
\begin{gathered}
{ }^{s} \underline{B}(t)=\underline{B}(t) \cdot \underline{\lambda}^{*}, \\
\underline{\lambda}^{*}=\lambda_{r}-{ }_{J} \lambda_{\varphi} .
\end{gathered}
$$

The air gap field ${ }^{a} \underline{B}$ excited by the armature current is given by

$$
{ }^{a} \underline{B}(t, I, \varphi)=\left(\begin{array}{c}
{ }^{p} \underline{B}\left(\sqrt{2} I e^{J\left(\omega_{s} t+0^{\circ}\right)}\right. \\
{ }^{p} \underline{B}\left(\sqrt{2} I e^{J\left(\omega_{s} t+120^{\circ}\right)}\right. \\
{ }^{p} \underline{B}\left(\sqrt{2} I e^{J\left(\omega_{s} t+240^{\circ}\right)}\right.
\end{array}\right) \cdot\left(\begin{array}{c}
e^{J\left(\phi_{q}+\psi+0^{\circ}\right)} \\
e^{J\left(\phi_{q}+\psi+120^{\circ}\right)} \\
e^{J\left(\phi_{q}+\psi+240^{\circ}\right)}
\end{array}\right)
$$

where ${ }^{p} \underline{B}$ is the normalized field due to one phase and can be also obtained by conformal mapping [6]. In (8) the angle $\phi_{q}$ defines the relative phase orientation to the quadrature axis of the machine, $\psi$ is the flux-weakening-angle and $\omega_{s}$ is the stator current angular frequency. We shall in the sequel systematically omit the coordinate $\Theta$ and the time argument $t$, and only indicate the dependency in $I$ and $\psi$ when applicable. We shall also label the quantities obtained by the conformal mapping approach with a CM exponent. The overall air gap field is thus defined as

$$
{ }^{g} \underline{B}^{\mathrm{CM}}(I, \psi)={ }^{a} \underline{B}^{\mathrm{CM}}(I, \psi)+{ }^{s} \underline{B}^{\mathrm{CM}} .
$$




\section{Leakage and nonlinearity}

The main idea of rewriting the CM governing equations sketched in section I is to obtain a CM formulation in which each parameter represents a physically motivated quantity in order to distinguish their origin within the electromagnetic field computation. The definition of the armature field ${ }^{a} \underline{B}$ in (8) includes an implicit formulation of $\underline{\lambda}$ in the ansatzfunction of the field ${ }^{p} \underline{B}$. For further purposes, $\underline{\lambda}^{*}$ must be factorized

$$
{ }^{g} \underline{B}^{\mathrm{CM}}(I, \psi)=\left(\underline{B}^{\mathrm{CM}}+{ }_{u}^{a} \underline{B}^{\mathrm{CM}}(I, \psi)\right) \cdot \underline{\lambda}^{*},
$$

where an arbitrary permeance state $\underline{\lambda}$ is identical for both field fractions of ${ }^{g} \underline{B}^{\mathrm{CM}}(I, \psi)$. In (10) the auxiliary field ${ }_{u}^{a} \underline{B}^{\mathrm{CM}}(I, \psi)$ is defined by

$$
{ }_{u}^{a} \underline{B}^{\mathrm{CM}}(I, \psi)={ }^{a} \underline{B}^{\mathrm{CM}}(I, \psi) \cdot\left(\underline{\lambda}^{*}\right)^{-1} .
$$

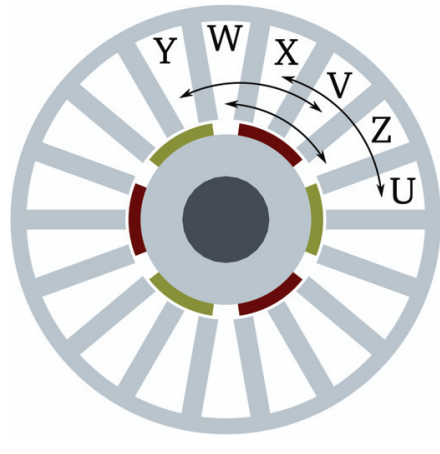

Fig. 1. SM-PMSM crosssection
Table 1. SM-PMSM parameters

\begin{tabular}{l|c}
\hline 3 & number of pole pairs \\
\hline 18 & number of stator teeth \\
\hline 0.73 & pole pitch factor \\
\hline $3 \mathrm{~mm}$ & permanent magnet height \\
\hline $24.5 \mathrm{~mm}$ & rotor radius (incl. PM) \\
\hline $0.8 \mathrm{~mm}$ & air gap height \\
\hline $54.2 \mathrm{~mm}$ & outer stator radius \\
\hline $43 \%$ & slot-opening-factor \\
\hline $1.244 \mathrm{~T}$ & remanence flux density \\
\hline $101 \mathrm{~mm}$ & length \\
\hline $4 \mathrm{~mm}$ & yoke width \\
\hline
\end{tabular}

\section{A) Ideal case}

The comparative study [7] for modern analytical models for the electromagnetic field prediction concludes that the relative error of the air gap flux density obtained by CM with respect to FE prediction increases strongly in function of the slot-opening to slot-pitch-ratio. In order to analyze this with CM field computation, a SM-PMSM with a slot-opening factor of $43 \%$ is studied. The cross-section of the motor is depicted in Figure 1. All parameters of the geometry and the electromagnetic evaluation are given in Table 1 . To evaluate the analytic field 

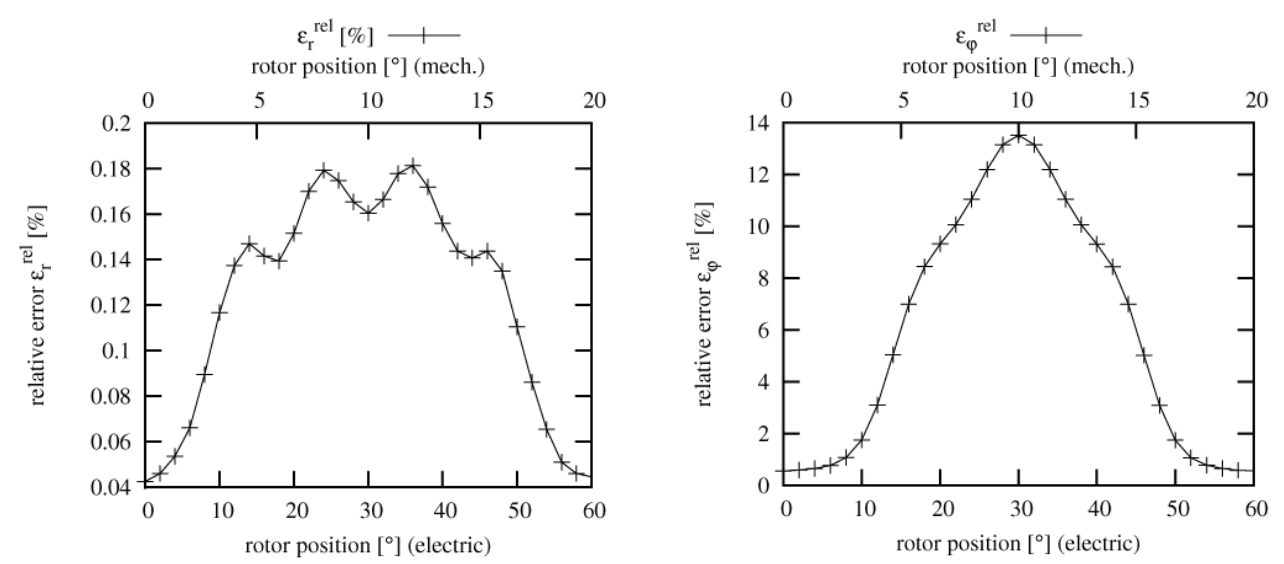

Fig. 2. Relative error of the radial and tangential flux density between IFE and CM in lo-load operation

computation according to (10), a quasi-static FE computation [8], where the stator and the rotor yoke are modeled as infinite permeable iron (IFE), utilizing Neumann boundary condition, is used as a reference simulation. Both methods (FEA, CM) are carried out with 180 rotational steps per electrical period.

Figure 2 shows the relative flux density error $\varepsilon^{\text {rel }}$, defined by

$$
\varepsilon_{x}^{r e l}=\left.\frac{\int_{0}^{2 \pi}\left(B_{x}^{\mathrm{CM}}-B_{x}^{\mathrm{IFE}}\right)^{2} d \theta}{\int_{0}^{2 \pi}\left(B_{x}^{\mathrm{IFE}}\right)^{2} d \theta}\right|_{x=r, \varphi}
$$

relating IFE and CM in radial and tangential direction in function of the rotor position. In the aligned position of magnets and teeth $\left(0^{\circ}, 60^{\circ}\right)$ the deviation in both components is minimal. The error strongly increases towards the critical positions, where the edges of the magnets are aligned with the edges of the teeth $\left(\sim 20^{\circ}, \sim 40^{\circ}\right)$. Between these positions, $\varepsilon^{\text {rel }}$ remains roughly on the same error level. Moreover, the magnitude of $\varepsilon_{\varphi}^{\text {rel }}$ is significantly higher than $\varepsilon_{r}^{r e l}$. This position dependent error characteristic can also be identified in the cogging torque diagram, given in Figure 3. The correlation of $T^{\mathrm{CM}}$ and $T^{\mathrm{FE}}$ follows the progression of the relative flux density error, cmp. Figure 2 , with exception of the natural point of symmetry $\left(30^{\circ}\right)$.

Due to the participation of both field directions to the resulting torque, defined according to the Maxwell stress theory by

$$
T=\frac{l r^{2}}{\mu_{0}} \int_{0}^{2 \pi} B_{r}(\Theta) B_{\varphi}(\Theta) d \Theta,
$$

where $l$ is the stack length and $\mathrm{r}$ the radius of the cylindrical integration surface, the tangential field mismatch of CM dominates the cogging-torque overestimation in Figure 3. 


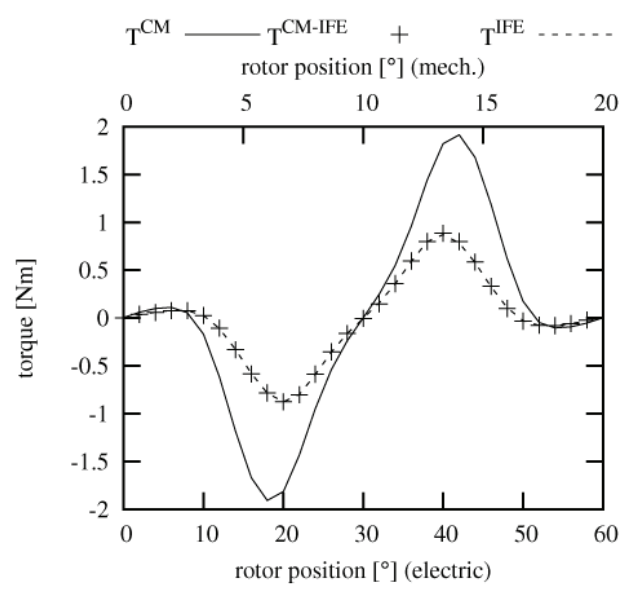

Fig. 3. Cogging-torque obtained by $\mathrm{CM}$,

IFE and CM-IFE in function of the rotor position of $6.67^{\circ}\left(20^{\circ}\right.$ electric $)$
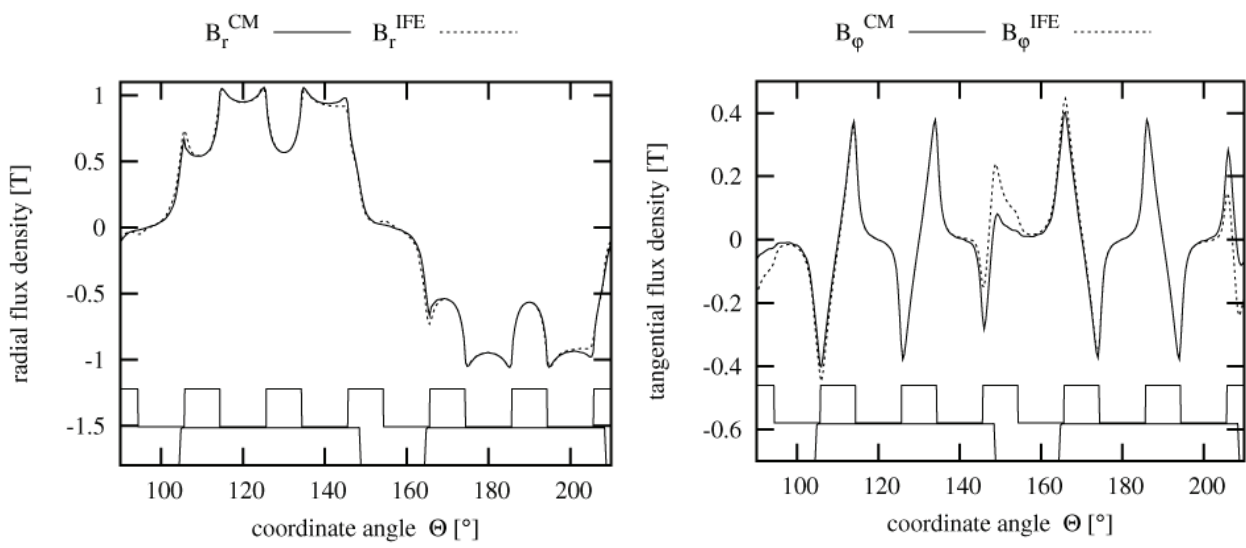

Fig. 4. Radial and tangential flux density in no-load operation in function of the coordinate angle $\Theta$ for a rotor position of $6.67^{\circ}\left(20^{\circ}\right.$ electric $)$

The flux density distribution in case of the critical position, aligning the edges of permanent magnets and teeth, depicted in Figure 4, shows a significant increase in its tangential component evoked by a flux path deformation in direction of the stator teeth. This effect is also known as slot leakage [9], and cannot be covered by the complex scalar quantity $\underline{\lambda}$ in (6). Adding this effect as optional

$$
{ }^{g} \underline{B}^{\mathrm{CM}-\mathrm{IFE}}(I, \psi)=\left(\underline{B}^{\mathrm{CM}}+{ }_{u}^{a} \underline{B}^{\mathrm{CM}}(I, \psi)+\Delta \underline{B}\right) \cdot \underline{\lambda}^{*},
$$

where $\Delta \underline{B}$ is the slot leakage of the rotor field. $\Delta \underline{B}$ can be determined by a single IFE computation in no-load case, leading to an identical flux density and therewith an identical cogging-torque progression, cf. Figure 3. A comparison of the torque results in rated load, overload and field-weakening operation in case of IFE and the application of (10) and (14) shows that the deviation of standard CM vanishes almost totally (see Fig. 5a, c, e). 


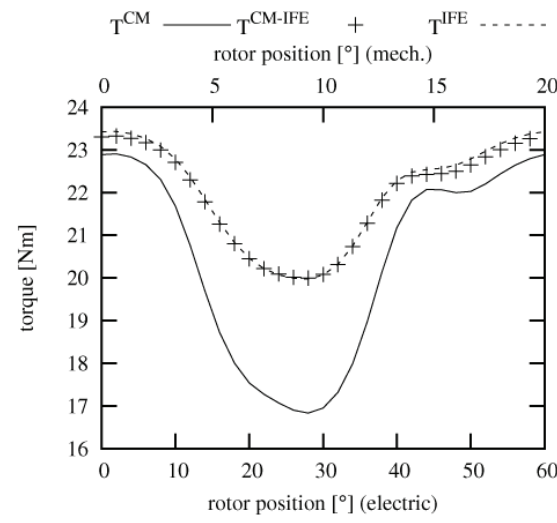

a) TD: $J=6 \mathrm{~A} / \mathrm{mm}^{2}, \psi=0^{\circ}$

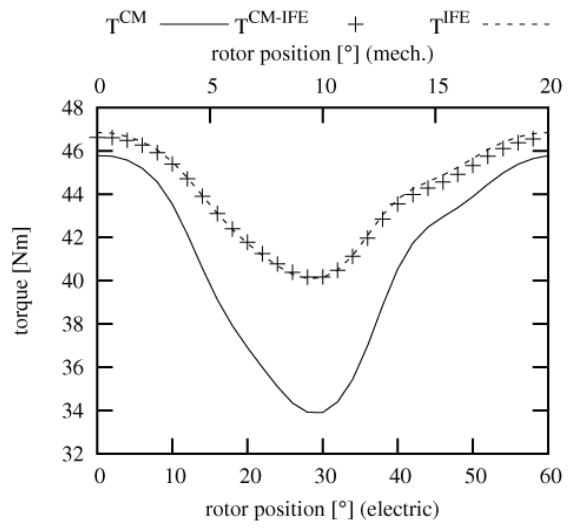

c) TD: $J=12 \mathrm{~A} / \mathrm{mm}^{2}, \psi=0^{\circ}$

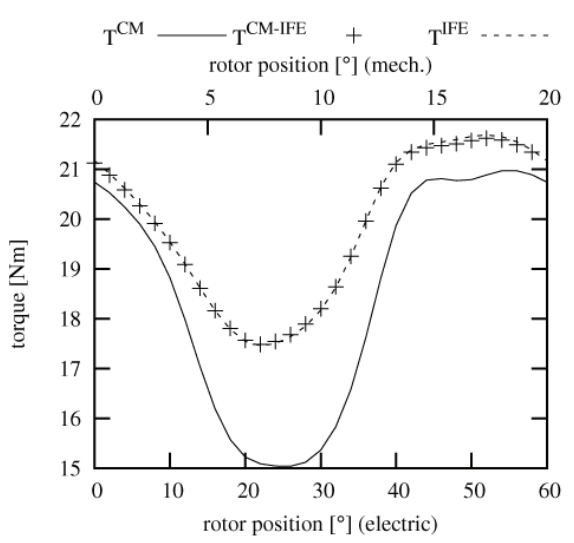

e) TD: $J=12 \mathrm{~A} / \mathrm{mm}^{2}, \psi=25^{\circ}$

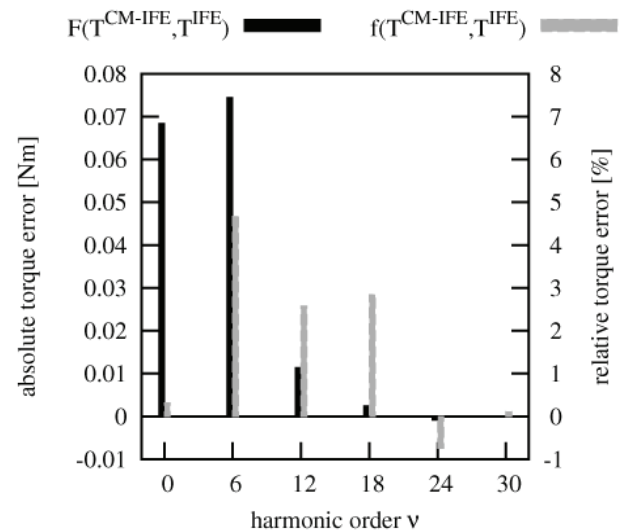

b) FD

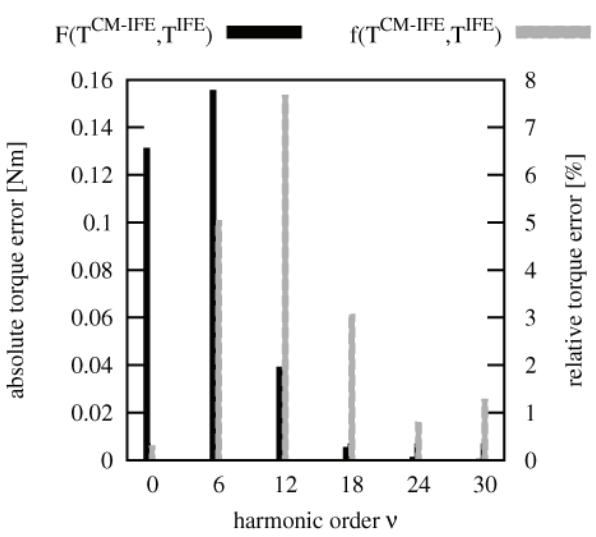

d) FD

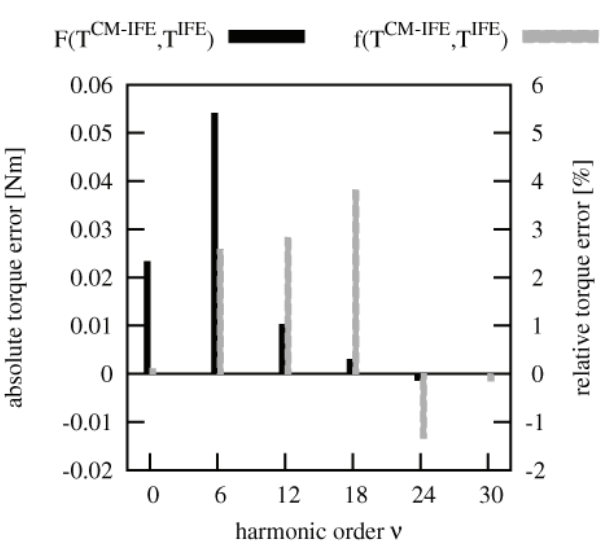

f) FD

Fig. 5. Comparison of torque computation in load, overloadand field-weakening operation obtained by CM, CM-IFE and IFE in time domain (TD) together with the relative and absolute torque error of CM-IFE and IFE in frequency domain (FD) 
The relative and absolute torque error between CM-IFE and IFE in frequency domain, denoted with the abbreviations $f$ and $\mathrm{F}$, is shown in Figure $5 \mathrm{~b}, \mathrm{~d}, \mathrm{f}$ ) for each operation point. The remaining minimal deviation originates from neglecting the relative permeability of the permanent magnets (PM), which is not included in the stator field representation of (8), and therefore not taken into account by the CM formulation according to (9).

\section{B. Nonlinear case}

The formalism in II-A to obtain (14) can also be applied in order to represent saturation. In this case, the permeance state $\underline{\lambda}$ turns from a constant quantity into a function of the magnitude of the current I and the flux-weakening-angle, yielding

$$
{ }^{g} \underline{B}^{\mathrm{CM}-\mathrm{IFE}}(I, \psi)=\left(\underline{B}^{\mathrm{CM}}+{ }_{u}^{a} \underline{B}^{\mathrm{CM}}(I, \psi)+\Delta \underline{B}\right) \cdot \underline{\lambda}^{*}(I, \psi) .
$$

For single points of interest, e.g. rated operation, $\underline{\lambda}(I, \psi)$ can be identified from a single nonlinear FE simulation ${ }^{g} \underline{B}^{\mathrm{FE}}(I, \psi)$ by

$$
\underline{\lambda}(I, \psi)={ }^{g} \underline{B}^{\mathrm{FE}}(I, \psi) \cdot\left[\left(\underline{B}^{\mathrm{CM}}+{ }_{u}^{a} \underline{B}^{\mathrm{CM}}(I, \psi)+\Delta \underline{B}\right)^{*}\right]^{-1}
$$

to further improve the analytic field computation.

Applying (16), $\underline{\lambda}$ also varies along $\Theta$ for each considered rotation angle. Figure 6 compares the relative permeances of (14),
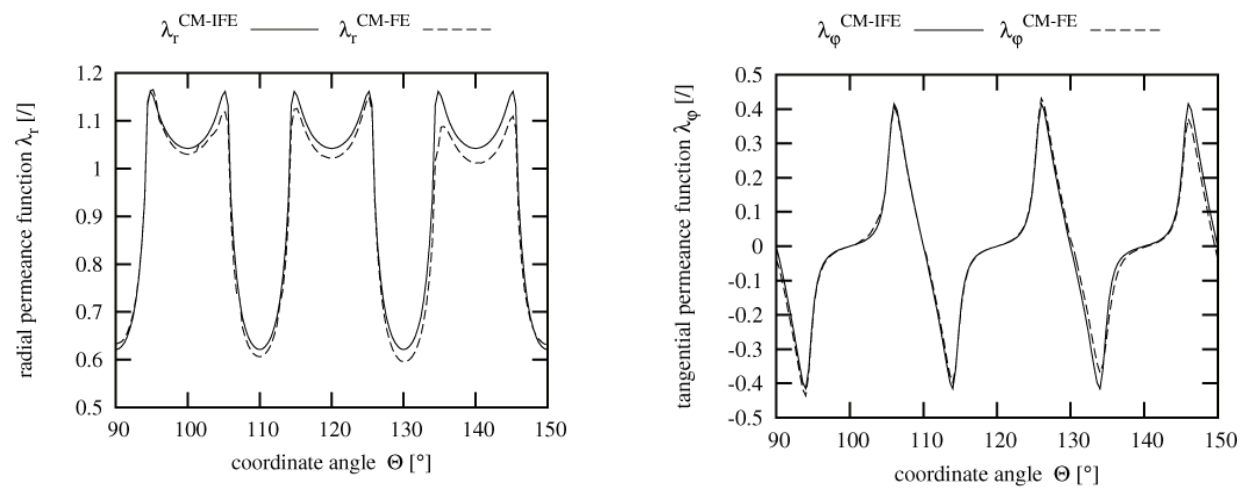

Fig. 6. Radial and tangential component of $\underline{\lambda}\left(J=6 \mathrm{~A} / \mathrm{mm}^{2}, \psi=0^{\circ}\right)$ in function of the coordinate angle $\Theta$ for a rotor position of $6.67^{\circ}\left(20^{\circ}\right.$ electric $)$

named $\underline{\lambda}^{\mathrm{CM}-\mathrm{FE}}$, and (15), denoted with $\underline{\lambda}^{\mathrm{CM}-\mathrm{FE}}$, for the critical position in case of rated operation $\left(J=6 \mathrm{~A} / \mathrm{mm}^{2}, \psi=0^{\circ}\right)$. The tangential curves are congruent, but one observes that $\lambda_{r}^{\mathrm{CM}-\mathrm{FE}}$ represents different saturation states for each tooth, whereas the progression of $\lambda_{r}^{\mathrm{CM}-\mathrm{FE}}$ since the permeance state is assumed identical.

The variation of $\lambda_{r}^{\mathrm{CM}-\mathrm{FE}}$ also considers the stator field modulation originating from the relative permeability of the PM, because (15) can not distinguish field effects separately. In 
consequence, unknown permeance states $\underline{\lambda}(\tilde{I}, \tilde{\psi})$ can be approximated by known values of $\underline{\lambda}(I, \psi)$, if and only if:

- the variable $\tilde{I}$ is in the neighborhood of I. Here, the permeance is assumed to be constant which is comparable to the frozen permeability technique [10]

- the flux-weakening-angle $\tilde{\psi}$ is identical to $\psi$. Elsewise the above mentioned side effects contained in $\underline{\lambda}(I, \psi)$, are assigned to wrong rotation angles.

Such an approximation in case of $80 \%$ and $120 \%$ of rated current density based on $\underline{\lambda}\left(J=6 \mathrm{~A} / \mathrm{mm}^{2}, \psi=0^{\circ}\right)$ has been applied to study the area of validity with respect to the load-torque computation according to (13). The comparison to nonlinear FEA and IFE in TD is depicted in Figure 7c, e. Due to the linearization of the permeance state, the curve $T^{\mathrm{CM}-\mathrm{FE}}$ is located above $T^{\mathrm{FE}}$ for $120 \%$ and below for $80 \%$. The curve progression is identical, with exception of an offset value. This conclusion is confirmed by the relative and absolute error evaluation shown in Figure $7 \mathrm{~d}$, $\mathrm{f}$, where the deviation of torque harmonics $(v \neq 0)$ is comparably low to the direct torque component $(v \equiv 0)$. In contrast to the ideal case, cmp. Figure 5 , the latter does not scale linearly with $J$, which is the effect of saturation. In order to show that (15) yields identical results on parameterized operation points, the torque evaluation in $\mathrm{TD}$ and $\mathrm{FD}$ is given in Figure 7a, b for rated operation.

\section{Further prospects}

The proposed approach, accounting for an analytic accurate field computation, yields to an accuracy comparable to FE-simulations. In case of ideal steel properties, the CM field computation captures all field effects, with the exception of the stator field deformation originating from the PM movement, over the whole operation range. Extending the radial correction term presented in [11] to an universal CM-operator would lead to an entire CM field representation for SM-PMSM. In case of nonlinear material properties, this paper demonstrates that parameterized permeance states can be used as approximations for other operation points in their neighborhood. In a further research, one can apply simple interpolations, e.g. a Spline of arbitrary order, to span a discrete state space over a large range of operations. Actually a test case with a large slot-openingfactor is in preparation, to compare measured quantities such as torque and induced voltage with values obtained by the CM method.

\section{Computational effort}

The field computation in CM formulation, relies on simple vector multiplications, independent from the level of detail. Since all ansatz functions are machine characteristics and are therefore constant for all operation points (with exception of $\underline{\lambda}(I, \psi)$, which is applicable in a range of operations), the simulation time lasts less than $1 \mathrm{~s}$ on a Single-Core processor. The quantities $\Delta \underline{B}$ and $\underline{\lambda}(I, \psi)$ are matrices, storing row vectors for all considered rotor positions, and do not slow down the computation. The quasi-static FE-simulations, performed 


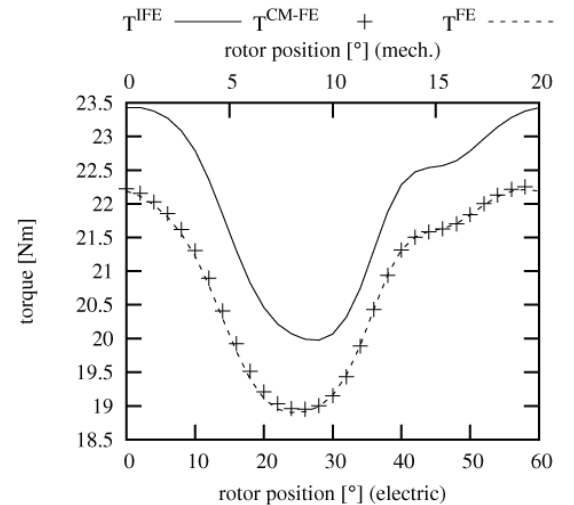

a) TD: $J=6 \mathrm{~A} / \mathrm{mm}^{2}, \psi=0^{\circ}$

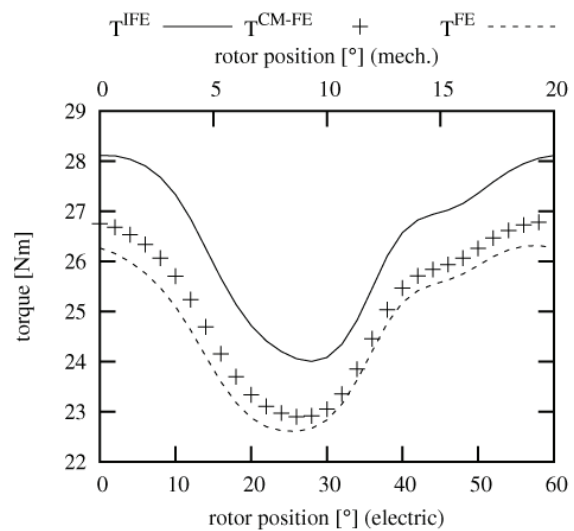

c) TD: $J=7.2 \mathrm{~A} / \mathrm{mm}^{2}, \psi=0^{\circ}$

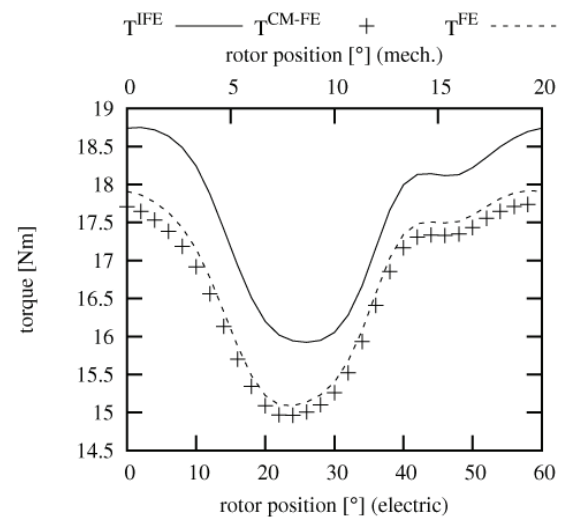

e) TD: $J=4.8 \mathrm{~A} / \mathrm{mm}^{2}, \psi=0^{\circ}$

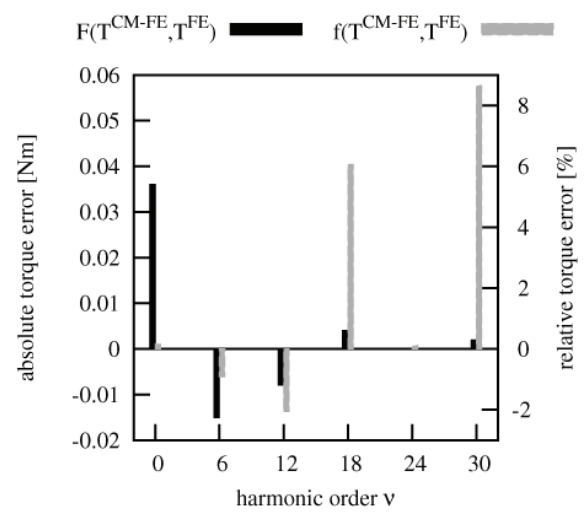

b) FD

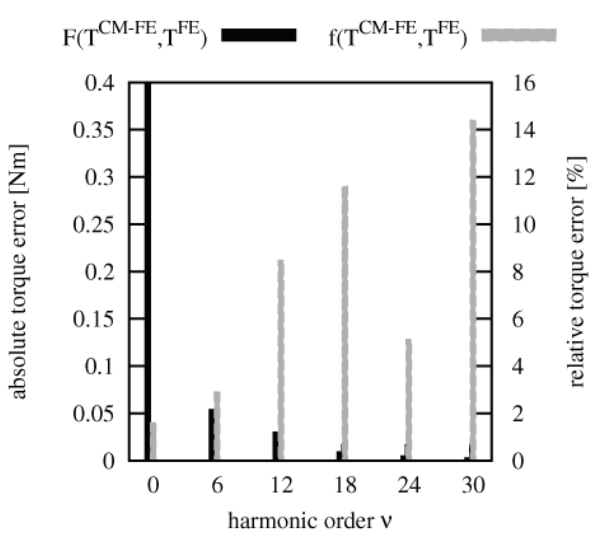

d) FD

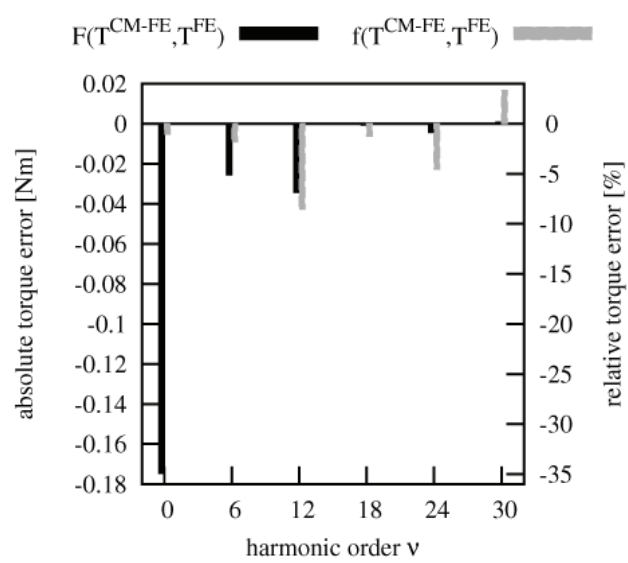

f) FD

Fig. 7. Comparison of torque computation in $80 \%, 100 \%$ and $120 \%$ of rated current density obtained by IFE, CM-FE and FE in time domain (TD) together with the relative and absolute torque error of CM-FE and FE in frequency domain (FD). The value of $\underline{\lambda}\left(J=6 \mathrm{~A} / \mathrm{mm}^{2}, \psi=0^{\circ}\right)$ is parameterized by (15). 
on a Quad-Core, to determine $\Delta \underline{B}$ in ideal case lasts 8 minutes (35541 DoF) and 39 Minutes for $\underline{\lambda}(I, \psi)(61118 \mathrm{DoF})$. Once computed, the presented CM extension yields an accuracy comparable to FEA still having such low computation times. This can be regarded as nearly real time capabilities. This speed up, together with its accuracy makes the proposed method attractive for different issues in a machine design process, e.g. design, minimization of torque or force harmonics or to study different field effects.

\section{Conclusion}

In this paper, the standard $\mathrm{CM}$ approach is extended to obtain a set of ansatz functions which can each be related to meaningful design quantities, modeling the effect of slotting and slot leakage on the rotor and armature field fraction respectively. This parameters can be reparameterized by ideal and nonlinear FEA to account for leakage and saturation phenomena. In ideal case (e.g. $\mu \rightarrow \infty$ ), one FE-simulation is sufficient to obtain an accuracy similar to FEA for the whole operation range including flux-weakening range. In nonlinear case, the standard permeance function has been extended to a permeance state in function of the current and the flux-weakening-angle to capture nonlinear phenomena. Each extracted permeance state is applicable in a range of operation points yielding results which are in good agreement to nonlinear FEA. Therewith, the proposed CM extensions allow to consider different levels of detail in the field simulation, which can be chosen in dependency of the intension to investigate such field problems. However, the proposed extension of classical CM has still the low computation time that is characteristic for such analytical models.

\section{References}

[1] Zhu Z., Howe D., Instantaneous magnetic field distribution in brushless permanent magnet DC motors. III. effect of stator slotting. Magnetics, IEEE Transactions on 29(1): 143-151 (1993).

[2] Zhu Z., Howe D., Chan C., Improved analytical model for predicting the magnetic field istribution in brushless permanent-magnet machines. Magnetics, IEEE Transactions on 38(1): 229-238 (2002).

[3] Hanselman D.C., Brushless Permanent Magnet Motor Design. (2nd ed.) The Writers' Collective (2003).

[4] Zarko D., Ban D., Lipo T.A., Analytical calculation of magnetic field distribution in the slotted air gap of a surface permanent-magnet motor using complex relative air-gap permeance. Magnetics, IEEE Transactions on 42(7): 1828-1837 (2006).

[5] Zarko D., Ban D., Lipo T.A., Fellow, IEEE Analytical solution for cogging torque in surface Permanent-Magnet motors using conformal mapping. Magnetics, IEEE Transactionson 44(1): 52-65 (2008).

[6] Binns K.J., Analysis and computaion of electric and magnetic field problems. Pergamon Press, New York (1963).

[7] Wu L., Zhu Z., Staton D. et al. Comparison of analytical models for predicting cogging torque in surface-mounted PM machines. [In:] Electrical Machines (ICEM), XIX International Conference on, 2010, pp. 1-6 (2010).

[8] van Riesen D., Monzel C., Kaehler C. et al. iMOOSE-an open-source environment for finiteelement calculations. Magnetics, IEEE Transactions on 40(2): 1390-1393 (2004).

[9] K. Oberretl, Die Berechnung des Streuflusses im Luftspalt von elektrischen Maschinen mit Käfigoder Dämpferwicklungen Teil 1: Theorie und Berechnungsmethoden. Archiv für Elektrotechnik Berlin 69(1): 11-22 (1986), compendex.

[10] Walker J.A., Dorrell D.G., Cossar C., Flux-linkage calculation in permanent-magnet motors using the frozen permeabilities method. IEEE Transactions on Magnetics 41(10): 3946- 3948 (2005).

[11] Zarko D., A systematic approach to optimized design of permanent magnet motors with reduced torque pulsations. Ph.D.-Thesis, University of Wisconsin, Madison (2004). 